\title{
Doctor of Medicine
}

National Cancer Institute

\section{Source}

National Cancer Institute. Doctor of Medicine. NCI Thesaurus. Code C39383.

The Doctor of Medicine degree is the first-professional postgraduate degree that requires completion of a program that meets all of the following criteria: completion of the academic requirements to begin practice in the profession; at least 2 years of college work prior to entering the program; and a total of at least 6 academic years of college work to complete a program of studies in any branch of medical science, including prior required college work plus the length of the professional program itself. The academic standard is equivalent to Ph.D. science. 